\title{
Through-focus or volumetric type of optical imaging methods: a review
}

Ravi Kiran Attota 


\title{
Through-focus or volumetric type of optical imaging methods: a review
}

\author{
Ravi Kiran Attota* \\ National Institute of Standards and Technology, Physical Measurement Laboratory, Engineering Physics Division, Gaithersburg, \\ Maryland, United States
}

\begin{abstract}
In recent years, the use of through-focus (TF) or volumetric type of optical imaging has gained momentum in several areas such as biological imaging, microscopy, adaptive optics, material processing, optical data storage, and optical inspection. We provide a review of basic TF optical methods highlighting their design, major unique characteristics, and application space. $\odot$ The Authors. Published by SPIE under a Creative Commons Attribution 3.0 Unported License. Distribution or reproduction of this work in whole or in part requires full attribution of the original publication, including its DOI. [DOI: 10.1117/1.JBO.23.7.070901]
\end{abstract}

Keywords: through-focus imaging; optical microscope; optical imaging; through-focus scanning optical microscopy; volumetric imaging; out-of-focus imaging; extended-depth-of-field imaging; axial scanning; three-dimensional imaging; bioimaging.

Paper 180203VR received Apr. 5, 2018; accepted for publication Jun. 11, 2018; published online Jul. 6, 2018.

\section{Introduction}

Considerable progress has been made in the area of optical microscopes and their applications during the past two decades. ${ }^{1-8}$ Optical tools with numerous variations and techniques have become a major part of research and development in the biorelated fields. Usage of through-focus (TF) optical imaging is steadily gaining momentum, particularly in biological applications. ${ }^{9-16}$ TF imaging is sometimes informally and interchangeably referred to using terms such as volumetric, out-offocus, blurred, defocused, extended-focused, extended-depthof-field, axial scanning, and three-dimensional (3-D) imaging.

Several uses of the TF optical image applications such as biological imaging, microscopy, adaptive optics, material processing, optical data storage, and optical inspection have been reported. ${ }^{17}$ In this paragraph, we highlight some specific applications and methods that use TF images. TF scanning optical microscopy (TSOM) makes use of a set of defocus optical images for 3-D shape metrology of target sizes ranging from sub-10 $\mathrm{nm}$ to over $100 \mu \mathrm{m}$, including nanoparticles, ${ }^{18,19}$ with subnanometer resolution. ${ }^{18-37}$ The ability to analyze optical illumination was also reported ${ }^{20}$ using the TSOM method. Much interest is given to two-photon (or multiphoton) microscopy that makes use of TF imaging to acquire 3-D volumetric data of biological samples, including brain tissue and bone calcium. ${ }^{11,14,15}$ High-speed TF imaging is used to track single-molecules in 3-D and observe their behavior during cell division, ${ }^{38}$ and it has also been used to image entire embryos. ${ }^{39}$ By simultaneously imaging different focal planes within the sample, it was possible to track the 3-D dynamics in live cells at high temporal and spatial resolution. ${ }^{12,13} 3$-D position, alignment, and orientation of submicroscopic features were made possible by TF polarization imaging in label-free as well as fluorescently labeled specimens. ${ }^{40}$ Cellular network dynamics such as spatiotemporal activity patterns in neuronal and astrocytic networks was demonstrated using TF imaging in

*Address all correspondence to: Ravi Kiran Attota, E-mail: ravikiran.attota@ nist .gov
3-D. ${ }^{14}$ 3-D automated nanoparticle tracking was demonstrated using TF images. ${ }^{41}$

\section{Significance of TF Image Collection}

Three important developments have increased the prevalence of TF imaging:

I. TF data conventionally include out-of-focus or blurred images. These blurred images were once considered to be of either inferior quality or not useful and were therefore mostly discarded, as in confocal microscopy. ${ }^{42}$ However, with the development of technology, improved optical simulations, and new insights, it was found that the out-of-focus TF images do contain useful information regarding the target being imaged. New applications and uses are being found for blurred optical images that were previously considered unusable. ${ }^{20,29,32,34,43-48}$ The major challenge was to extract high quality, usable information from the blurred TF optical images. $\mathrm{TSOM}^{18-37}$ is an example of a method where useful 3-D shape information can be extracted from a set of TF, blurred, optical images. TSOM enables 3-D shape metrology with subnanometer measurement resolution using a conventional optical microscope. ${ }^{24,25}$ TSOM has demonstrated the capability of extracting 3-D shape information of targets ranging in size from sub-10 nm to $>100 \mu \mathrm{m}$, with measurement resolution comparable with scanning electron microscopy or atomic force microscopy.

II. The second development is the advent of new applications that require collection of TF or volumetric data. Examples include high-speed 3-D tracking of nanoparticles, ${ }^{38,41}$ observation of high-speed cell division $^{38}$ and cellular network dynamics in $3-\mathrm{D},{ }^{14}$ and 3-D volumetric data of biological samples such as brain tissue and bone calcium. ${ }^{11,14,15}$ 
III. The third development is that advances in technology, instrumentation, simulations, and computation have enabled collection of TF images with sufficiently high quality and at sufficiently high speed to allow TF information to be readily utilized for many applications. For example, the development of objective lens scanning using piezoscanners has made it possible to readily adopt any conventional optical microscope into a high-speed TF image collection tool with acquisition times as small as $200 \mathrm{~ms}^{41}$ Optical tool developments have also enabled the simultaneous collection of TF images, with no scanning parts. ${ }^{38-40}$

It is now firmly established that TF optical images are beneficial for many applications. A survey of prior work reveals that many optical methods for TF image collection have been developed. It would be impossible to describe or even mention all of them in this review. However, most of the methods that have been published are variations of a few unique or basic methods. We here review the TF methods that represent the foundation of most published techniques.

\section{Through-Focus Image Collection Methods}

In this section, we describe most of the basic TF methods that have been published. These methods are depicted using simplified schematics and brief summaries. Note that in the following figures, the portions of the diagrams enclosed using dashed boxes indicate components or aspects of the technique that are unique to that particular method. Note also that some figures (e.g., Fig. 1) show multiple interrelated techniques.

\subsection{Scanning the Sample Stage Along the Focus Axis}

Sample stage scanning, shown schematically in Fig. 1(a), is the classic and the most widely used method to obtain TF images. It is simple and straightforward and nearly every optical microscope has some provision to adjust the sample along the focus axis: these methods range from manual focusing on basic instruments to more sophisticated motorized or piezoscans on high-end modern tools. The scanning range can be up to several tens of millimeters, and positional accuracy can be better than $10 \mathrm{~nm}$. However, TF scan speeds are typically regarded as relatively slow. Depending on the focus scan range, the exposure time, and the number of focal steps required, the total scan time could extend up to several minutes. The focus accuracy of this TF technique can be among the best, with high reproducibility and low distortion. However, optical mechanical instability ${ }^{32}$ and illumination aberrations ${ }^{49,50}$ have also been reported for such systems.

TF optical images collected by the stage-scanning method can be stacked at their respective focus positions to create a 3-D space filled with the optical intensities. Plotting the optical intensities in a vertical cross-section through this 3-D space results in a TSOM image [Fig. 1(e)]. Even though TSOM uses conventional optical images (i.e., not a resolution enhancement method), it provides subnanometer 3-D shape measurement resolution ${ }^{24}$ as it uses additional information present in the out-of-focus optical images. TSOM images are sensitive to changes in the (i) 3-D shape of a target, ${ }^{24,29}$ (ii) position of a target in a $3-\mathrm{D}$ space, ${ }^{41}$ (iii) optical properties of a target, and (iv) illumination. ${ }^{20} \mathrm{~A}$ differential TSOM image (pixel-by-pixel
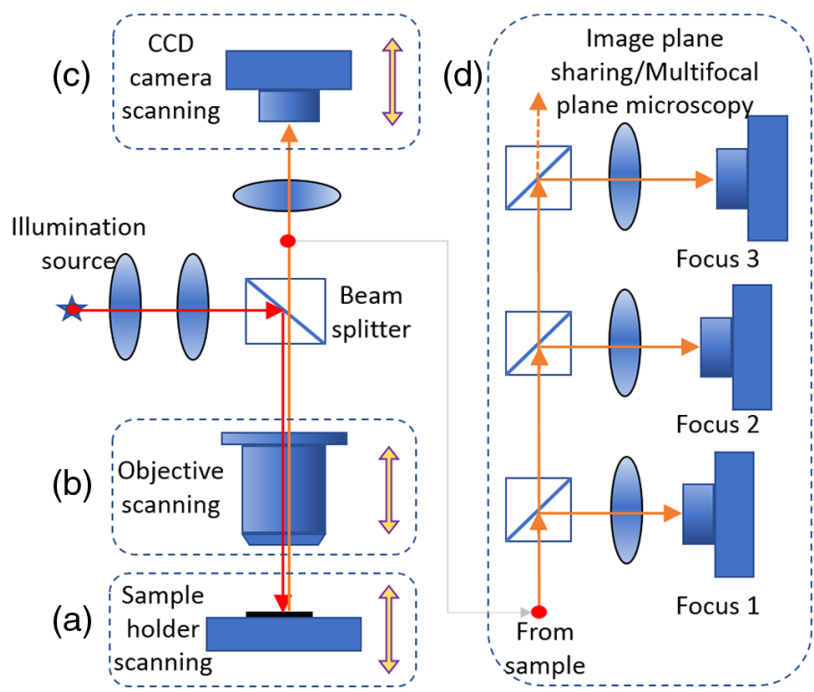

(e)

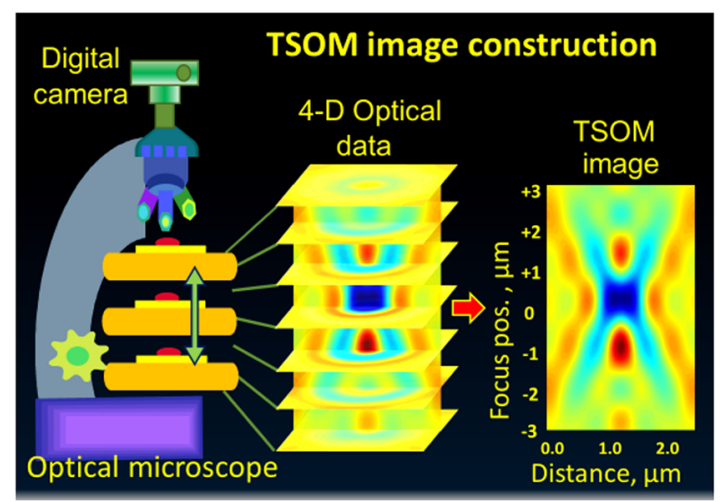

Fig. 1 TF image acquisition methods schematically shown using reflection (epi-illumination) optical microscopes. The methods include axial scanning of (a) the sample holder, (b) the objective lens, or (c) the camera; (d) splitting the imaging beam and focusing it onto multiple cameras set to different focal positions. (e) A typical TF scanning optical microscopy image construction using stage-scanning method (Video 1, MPEG, 0.5 MB [URL: https://doi.org/10.1117/1 .JBO.23.7.070901.1).

difference between two well-aligned TSOM images) highlights all these differences with much higher signal-to-noise ratio compared to a conventional best-focus, top-down optical image. ${ }^{18,19}$ TSOM is a strong candidate to analyze the position, shape, and optical properties of soft nanoparticles (e.g., hydrogel nanoparticles) in their native liquid environment, in addition to hard nanoparticles. ${ }^{41}$

\subsection{Scanning the Objective Lens Along the Focus Axis}

Another method of collecting TF images is by scanning the objective lens along the focal axis [Fig. 1(b)], generally using piezomotors. ${ }^{14,41,51}$ A relatively high-speed TF image collection time of $200 \mathrm{~ms}$ has been reported ${ }^{41}$ using this method, which makes it suitable, e.g., for nanoparticle tracking in 3-D space. It is relatively easy to convert a conventional microscope into a TF image collecting tool by replacing its objective lens base with one of the several commercially available objective-scanning piezomotors. In this high-speed scanning mode, the image quality could be degraded. However, this approach 
has the advantage of not disturbing the specimen because the sample stage remains stationary during imaging. Mechanically, this method is more stable than the stage-scanning method.

\subsection{Scanning the Image Plane Along the Focus Axis}

Through focus image collection can also be accomplished by scanning the image plane or camera, ${ }^{12,32}$ as shown in Fig. 1(c). The same effect can also be achieved by scanning a replica of the image plane. ${ }^{52}$ This method is reported to have the advantage of avoiding the spherical aberration common to other optical refocusing systems; it also allows for fast TF scans, extending the working distance, and keeping the specimen on stage stationary and undisturbed. ${ }^{52}$

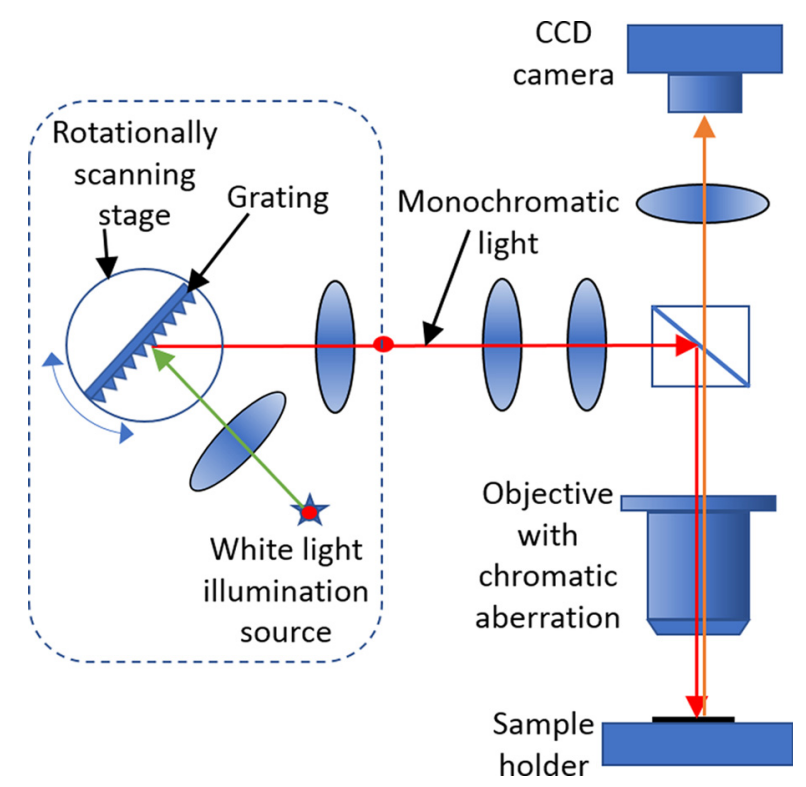

Fig. 2 TF image collection configuration by wavelength-scanning using an objective with chromatic aberration. In the setup shown here, the white light is split into different wavelengths using a rotationally scanned grating.

\subsection{Multifocal Plane Microscopy}

Instead of scanning the image plane, one alternative is to split the imaging beam into several fixed beams that are refocused simultaneously onto multiple cameras positioned at different focal distances, ${ }^{12,13,32}$ as shown in Fig. 1(d). This is also called image plane sharing microscopy. ${ }^{32}$ It has the main advantage of avoiding scanning altogether (of stage, objective or imaging plane). In this configuration, the $\mathrm{TF}$ images are collected simultaneously that enables high-speed TF image collection. However, since the imaging beam is split into several beams, the available optical intensity at each camera is reduced, which may lower the signal-to-noise ratio of the optical data, unless the collection time is increased. The number of focal planes available is also limited to the number of times the imaging beam is split. Since there are no moving (scanning) parts, this is one of the most mechanically stable configurations.

\subsection{Wavelength Scanning Method}

For microscope objectives with chromatic aberration, differing wavelengths will have different focus positions. Normally microscope objectives are designed to minimize chromatic aberration. However, chromatic aberration can also be used to obtain TF images. ${ }^{32,34,53}$ In this method, both the chromatic aberrated objective and the sample stage are kept at fixed positions. Instead, the wavelength of illumination is varied (or wavelength-scanned) so each wavelength focuses at a different focus position on the sample, enabling TF image collection (Fig. 2). A variation of this approach, which utilizes the chromatic dependence of diffraction, has been developed using tricolored LED illumination and a spatial light modulator (SLM) ${ }^{53}$ Since the position of sample stage is not scanned, the wavelength scanning approach generally has superior mechanical stability compared to conventional stage-scanning optical microscopes. It also allows high-throughput TF image collection due to the ability to quickly and precisely tune the wavelength $^{34}$ (faster than scanning a stage).

\subsection{Flexible-Membrane Liquid Lens}

While there are many variations in design and implementation, the general concept of the approach shown in Fig. 3(a) is that the fluid pressure inside two flexible membranes is varied to make

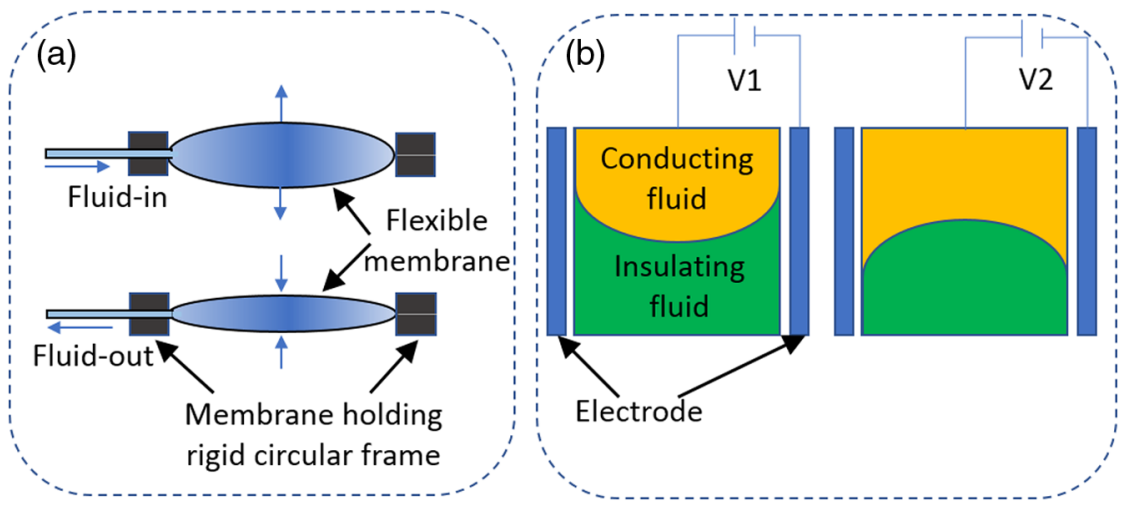

Fig. 3 Focus-tunable lenses. (a) Flexible membrane liquid lens. The effective focal length changes due to changes in the curvature of the flexible membranes when the pressure inside is varied. (b) Liquidtunable lens. The curvature at the interface of two immiscible liquids varies due to changes in the interfacial tension when the applied voltage across the insulating and conducting fluids is changed. The change in the curvature results in varying the focal length. 
the membranes inflate or deflate. This process alters the radius of curvature of the membranes and thus changes the effective focal length of the lens, allowing TF image collection. ${ }^{54-62}$ The membrane curvature can alternatively be varied by different mechanisms such as changing the aperture diameter thus squeezing the membranes. ${ }^{63}$ These lenses are generally fast, but are prone to have optical aberrations. ${ }^{56}$

\subsection{Liquid-Tunable Lens}

This method, shown in Fig. 3(b), is also known as a variable focus liquid lens. These lenses utilize refraction at a liquidliquid boundary interface. Focal adjustment is achieved by using a variable voltage to tune the curvature at the boundary interface of two immiscible liquids. A spherical surface is formed at the boundary of a polar and an apolar liquids. The curvature of the interface can be controlled by adjusting the relative wettability through electro-wetting. ${ }^{15}$ This creates a lens with variable focal length to enable TF image collection. ${ }^{15,55,63,64}$ Liquid lenses are relatively fast and very durable and exhibit a high degree of phase shift, i.e., focal length change. These lenses have a long functional life and low sensitivity to mechanical stress, and they are also advantageous for autofocus applications due to their relatively low power consumption. Liquid-tunable lenses have relatively low cost and are commercially available. However, the optical performance of liquid-tunable lenses suffers from significant spherical as well as higher-order, gravity-induced aberrations. ${ }^{56}$

\subsection{Adaptive Optics}

As shown in Fig. 4, adaptive optical elements such as a tip/tilt or deformable mirror can be inserted into the optical path of a microscope to enable TF image collection. ${ }^{32,65,66}$ Common examples of adoptive optics elements are deformable mirror devices or liquid crystal SLMs. The adoptive optics is used to alter the phase of incident wave fronts to displace the focal spot. Tip/tilt applied to the adoptive optics shifts the focus laterally within the focal plane, while defocus translates along the optic axis. ${ }^{65}$ In the implementation with a tip/tilt mirror, a basic closed-loop quad cell is used to control the mirror. A high-order deformable mirror with a Shack-Hartmann sensor is

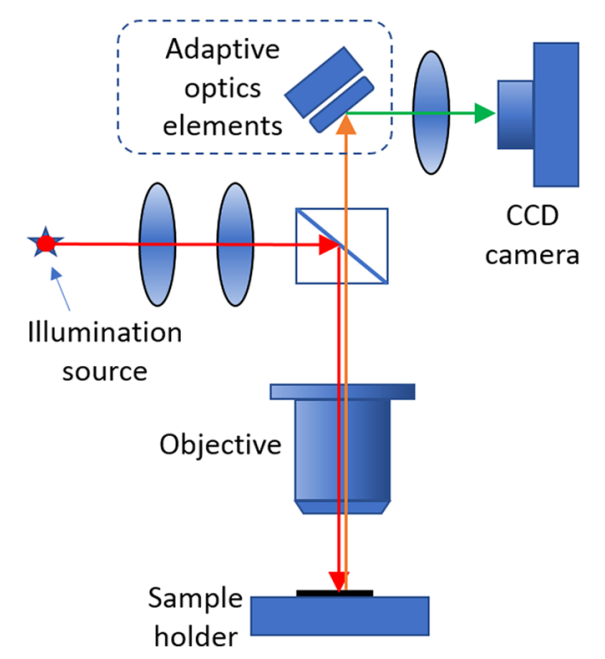

Fig. 4 Schematic showing a design of using adaptive optical elements for TF image collection using tip/tilt or deformable mirrors. used in the deformable mirror implementation. This approach can also correct high-order residual aberrations as well as performing the TF scanning without $z$-axis movement. A key advantage of these methods is high-speed TF image collection $(\sim \mathrm{KHz})$. Adaptive optics technology and hardware are readily available and are most commonly used for biological applications. $^{16}$

\subsection{Multifocus Microscopy}

Multifocus microscopy (MFM) allows rapid and simultaneous acquisition of TF images using a single exposure (Fig. 5). Unlike a conventional microscope, there are no scanning parts. ${ }^{38-40}$ This is achieved by placing a multifocus grating (MFG) at the Fourier plane followed by a chromatic correction grating (CCG) and prism [Fig. 5(a)]. The combination of these elements divides the primary image into several TF images and simultaneously projects them onto the plane of the CCD camera. Unlike a conventional microscope, the CCD camera in MFM is divided into several squares, each one of which is used to collect one of the focal plane images [Fig. 5(b)]. To date, MFM has been used primarily for biology applications, and this technology is not yet widely available.

\subsection{Aperture-Scanning Fourier Ptychography}

In this method, an aperture is placed at the Fourier plane as shown in Fig. 5(c), and it is scanned in a raster manner while simultaneously collecting intensity images of the object. The acquired images are then synthesized in the frequency domain to recover the complex hologram of extended objects. This can then be digitally propagated into different planes along the optical axis to extract TF images of the object. ${ }^{67}$ An alternative method is to scan the camera instead of an aperture. ${ }^{67}$

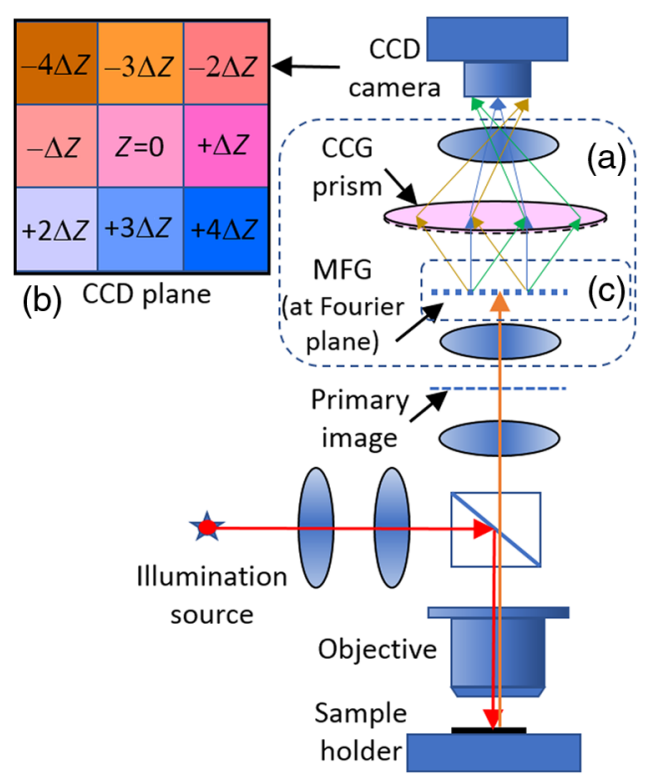

Fig. 5 Simplified schematic of MFM. (a) Optical elements unique to MFM. MFG, multifocus grating located at the Fourier plane; CCG, chromatic correction grating. (b) Images at predetermined focal planes are collected simultaneously by dividing the camera area as shown here. Optical designs are available to collect $3 \times 3$, $4 \times 4$, or $5 \times 5$ images in the camera. (c) The location of aperture for aperture-scanning Fourier ptychography method. 
Because of the requirement to scan certain parts, this approach could be slower.

\subsection{Confocal Microscopy}

In confocal microscopy, out-of-focus image information is selectively discarded by placing a pin hole in front of the camera (Fig. 6). This allows collection of image-slices that contain primarily in-focus image information. By scanning the sample along the focal axis, multiple such image-slices through the sample are collected, and this enables a 3-D reconstruction of the sample surface. Numerous alternative implementations and variations on this basic method of data acquisition have been published. This is one of the most widely used TF imaging methods, particularly in biology.

\subsection{Light Sheet Microscopy}

In light sheet microscopy (LSM), the sample (usually transparent or semitransparent) is illuminated from the side using a thin sheet of light. Typically, the light source is rigidly coupled to the objective and aligned with its focal plane (Fig. 7) ${ }^{68-70}$ By scanning the sample stage along the focal axis, volumetric images of the specimen can be obtained. In a strict sense, this method is not a TF method because the imaging plane is always in focus. However, it can extract 3-D volumetric image information similarly to a confocal microscope. Unlike a confocal microscope, however, the out-of-focus image information is not discarded, it is simply not illuminated. Numerous variations of this basic technique have been published. ${ }^{69,71}$ LSM is mainly used to study biological tissues. Because of the opacity of such tissues, 3-D imaging is typically limited to a depth of 500 to $1000 \mu \mathrm{m} .^{72,73}$ However, in some studies, LSM appears to outperform confocal microscopy. ${ }^{10}$

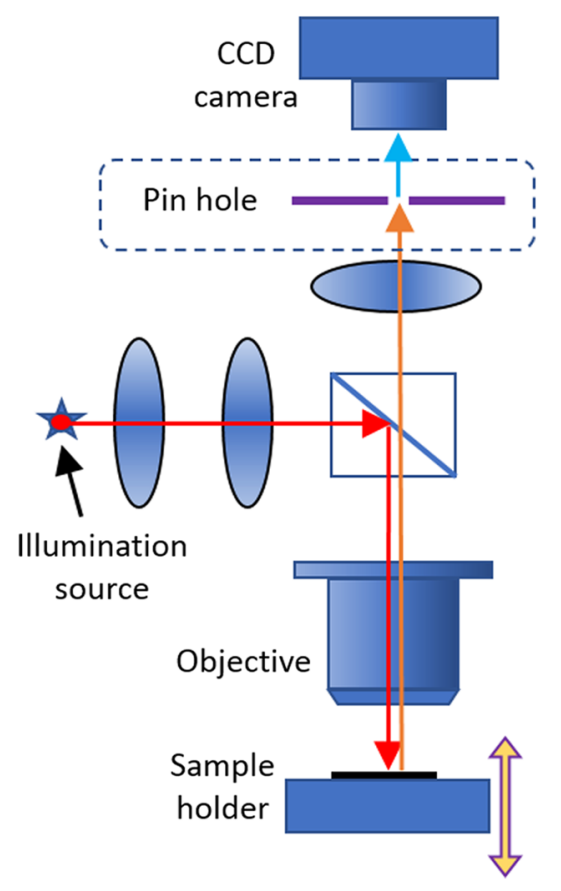

Fig. 6 Basic schematic of a confocal microscopy setup. The pin hole selectively discards out-of-focus images information.

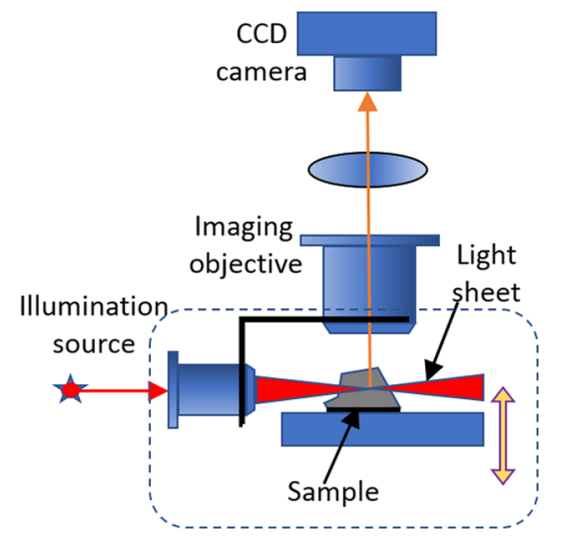

Fig. 7 Simplified schematic of LSM. In the basic setup, the imaging and illuminating objectives are rigidly coupled. The sample is scanned along the focal axis to collect volumetric images.

\subsection{Light Field Microscopy}

In this microscopy, a microlens array is inserted into the optical train of a conventional microscope between the main lens and sensor plane, and this enables the capture of light-fields of specimens in a single image (Fig. 8). ${ }^{7,75} \mathrm{TF}$ images can then be extracted through application of 3-D deconvolution to this single image. LFM also has the capability to extract different perspective views from the single image. In an LFM, spatial resolution is determined by the number of microlenses. A disadvantage of LFM is the trade-off between spatial resolution and angular resolution. $^{74}$

\subsection{Phase Retrieval Techniques}

Phase retrieval techniques make use of the relationship between phase and propagation direction in an optical microscope. This method initially requires a few TF images that are inverted to recover phase and amplitude quantitatively. By making use of the retrieved phase and the amplitude, the entire set of TF images can then be calculated. ${ }^{76-78}$ A variation of this method, which is sometimes called quantitative phase imaging, has found many useful applications in biology. ${ }^{79}$

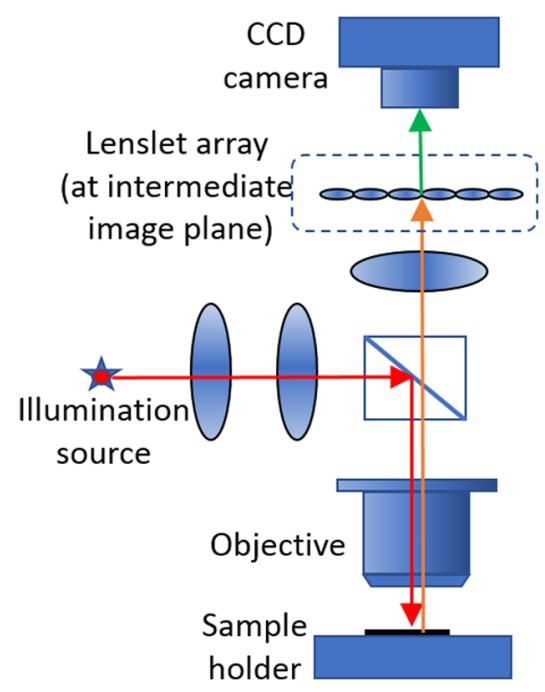

Fig. 8 Simplified schematic of LFM. The lenslet arrays are located at the intermediate image plane. 


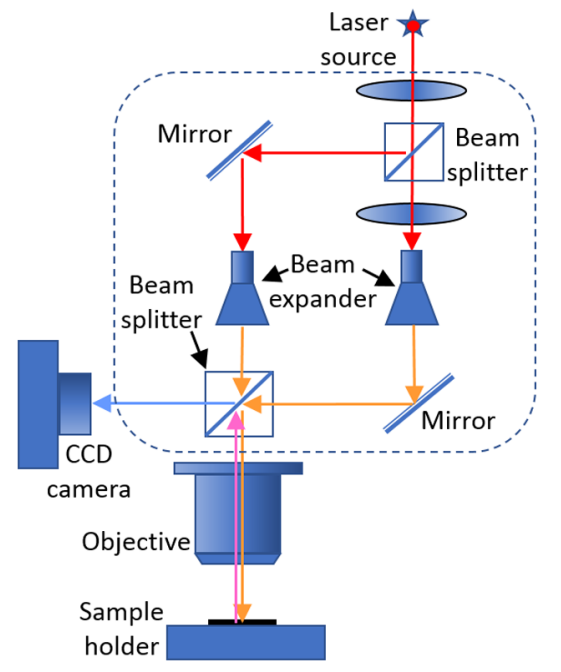

Fig. 9 Simplified schematic of DHM.

\subsection{Digital Holography Microscopy}

Holographic microscopy (including digital holography) is a coherent imaging system, and its advantage lies in the instantaneous and quantitative acquisition of both amplitude and the phase information from the reconstruction of the wavefront. ${ }^{80-86}$ Digital holography microscopy can numerically extract TF images from a single experimentally recorded hologram without the need to move the sample (Fig. 9). The digital holographic approach has been successfully implemented to speed up TSOM image acquisition. ${ }^{31}$ Since no mechanical scanning is involved, and a complete set of TF images can be extracted from a single holographic image, it is a high-speed TF imaging method.

\subsection{Other Methods}

Acousto-optic lens scanning ${ }^{17}$ can achieve a pure focal scan at a very high speed $(\approx 400 \mathrm{kHz})$. This is accomplished using two adjacent counterpropagating acoustic waves with a synchronized frequency chirp so the transverse scans subtract to cancel each other, whereas the focal scans add. Acousto-optic modulators and acousto-optic scanners are used along with a laser beam, and there is a 180-deg phase shift between the two frequency-modulating signals. The light is focused on a CCD camera that is mounted on a translation stage and used to measure the focal distance. Temporal and spatial focusing ${ }^{87}$ achieves high TF speeds with no moving parts. Full-field optical coherence microscopy produces volumetric imaging with all in-focus images similar to confocal microscopy. ${ }^{88,89}$ It is based on the spatial coherence gate principle and generates in parallel complete two-dimensional TF type images without scanning.

Many variations of the preceding general TF methods have also been published. Some are a combination of two (or more) TF methods with some other methods. For example, a combination of high speed sinusoidal vibration of the microscope objective along with "smart" movements of galvanometric $x-y$ scanners (to repeatedly scan the laser focus along a closed 3-D trajectory) enables high-speed acquisition of TF images for two-photon microscopy that permits fast fluorescence measurements. $^{14}$
Optical images such as the TSOM images [Fig. 1(e)] can be constructed using any one of the TF method presented here. However, for the TF methods such as confocal and lightsheet microscopy, all the images are in-focus creating a different type of TSOM image.

\section{TF Images Categorization}

\subsection{Optical Data Type}

TF optical images can be broadly divided into three types depending on the type of optical data

I. In the first type, the set of TF images usually contains a best focus image, along with many out-of-focus (or blurred) images on the either sides of the best focus image. A typical example for this type of TF data collection is the conventional stage-scanning method. ${ }^{37}$

II. In the second type, nearly all the TF images are either in-focus or contain only in-focus image information enabling one to visualize or reconstruct the 3-D volume of the sample. Confocal microscopy ${ }^{42,90}$ is a typical example for this kind of TF data collection method. Some TF methods are able to extract both the first and the second types of TF optical data such as the digital holography ${ }^{82}$ method.

III. The third type, which is often referred to as either super-resolution microscopy ${ }^{4}$ or nanoscopy, ${ }^{1}$ is a highly localized imaging method with measurement resolution down to tens of nanometers. In this stochastic approach, only a small subset of molecules is switched on in a given 3-D volume at any particular moment in time using fluorescence principle while the majority remains in a nonfluorescent "dark" or "off" state. ${ }^{91}$ Super-resolved images are reconstructed from the positions of thousands to millions of single molecules that have been recorded in thousands of camera frames. ${ }^{92}$ Several thorough review articles have been published on this subject, ${ }^{1-5}$ and hence no further discussion of this type of TF imaging is included in this review.

\subsection{Scanning}

Depending on the presence or absence of scanning, TF image collection methods can be divided into two groups.

I. In the first group, the $\mathrm{TF}$ image collection involves scanning (or continuous variation) of some parts of the optical microscope. Typical examples include objective scanning and light-sheet microscopy. Even though the phase retrieval technique does not require scanning, it still requires at least two images at different focal planes.

II. In the second group of methods, no scanning is involved to acquire TF images. MFM and digital holography microscopy are typical examples of this group. 
Table 1 Complete categorization of the TF scanning methods.

\begin{tabular}{|c|c|c|c|c|c|c|}
\hline $\begin{array}{l}\text { Section } \\
\text { no. }\end{array}$ & TF method & TF image data includes & Scanning & $\begin{array}{l}\text { Scanning } \\
\text { method }\end{array}$ & $\begin{array}{l}\text { Image } \\
\text { extraction }\end{array}$ & Best speeds ${ }^{a}$ \\
\hline 3.1 & Stage scanning & In-focus and out-of-focus & Yes & Stage & Direct & A few hundred ms \\
\hline 3.2 & Objective scanning & In-focus and out-of-focus & Yes & Objective & Direct & A few hundred ms \\
\hline 3.3 & Image plane scanning & In-focus and out-of-focus & Yes & Camera & Direct & A few hundred ms \\
\hline 3.4 & Multifocal plane microscopy & In-focus and out-of-focus & No & NA & Direct & High-speed/instantaneous ${ }^{b}$ \\
\hline 3.5 & Wavelength scanning & In-focus and out-of-focus & Yes & Wavelength & Direct & A few hundred ms \\
\hline 3.6 & $\begin{array}{l}\text { Flexible-membrane liquid } \\
\text { lens }\end{array}$ & In-focus and out-of-focus & Yes & Focus & Direct & A few ms \\
\hline 3.7 & Liquid-tunable lens & In-focus and out-of-focus & Yes & Focus & Direct & A few ms \\
\hline 3.8 & Adaptive optics & In-focus and out-of-focus & Yes & Focus & Direct & A few ms \\
\hline 3.9 & MFM & In-focus and out-of-focus & No & NA & Direct & High-speed/instantaneous $^{b}$ \\
\hline 3.10 & $\begin{array}{l}\text { Aperture-scanning Fourier } \\
\text { ptychography }\end{array}$ & In-focus and out-of-focus & Yes $^{\mathrm{c}}$ & NA & Computational & Tens of seconds ${ }^{d}$ \\
\hline 3.11 & Confocal microscopy & In-focus only & Yes & Stage & Direct & A few hundred $\mathrm{ms}$ \\
\hline 3.12 & LSM & In-focus only & Yes & Stage & Direct & A few hundred ms \\
\hline 3.13 & LFM & In-focus and out-of-focus & No & NA & Computational & $\begin{array}{l}\text { High-speed/ } \\
\text { instantaneous }^{\text {b d }}\end{array}$ \\
\hline 3.14 & Phase retrieval techniques & In-focus and out-of-focus & Yes/no ${ }^{e}$ & Any ${ }^{e}$ & Computational & $\begin{array}{l}\text { High-speed/ } \\
\text { instantaneous }^{\text {b d }}\end{array}$ \\
\hline 3.15 & $\begin{array}{l}\text { Digital holography } \\
\text { microscopy }\end{array}$ & In-focus and out-of-focus & No & NA & Computational & $\begin{array}{l}\text { High-speed/ } \\
\text { instantaneous }^{\text {b d }}\end{array}$ \\
\hline
\end{tabular}

NOTE: NA, not applicable.

aApproximate values to collect a set of TF images.

${ }^{\text {b}}$ Either only one image or several images simultaneously are collected.

'Scanning of an aperture.

dPlus computational time.

eEither scanning or nonscanning methods can be used to obtain a few images at different focal positions.

\subsection{Image Extraction}

Depending on the image extraction method, two groups of TF approaches can be formed:

I. Some TF methods enable direct image acquisition, such as stage scanning, liquid-tunable lens, etc.

II. Some TF methods require indirect extraction (or computational extraction) of TF images. Phase retrieval and digital holographic microscopy are typical examples for this group.

A complete categorization of all the TF methods is presented in Table 1.

\section{Speed and Quality of TF Image Collection}

One of the main challenges in modern optical microscopy is fast and sensitive acquisition of TF data. ${ }^{11,38}$ In some applications, such as in single molecule tracking methods, ${ }^{38,41}$ it is essential to have a high-speed TF image collection. In contrast, for certain reference metrology applications, high-speed collection may not be necessary, but high precision is of paramount necessity. ${ }^{24,25}$ In the following sections, we discuss relative speeds and quality of TF image collection methods.

\subsection{Speed}

The speed of TF image collection and image quality will usually trend in opposite directions. In this review, the image quality mostly refers to signal-to-noise ratio. Typically, higher collection speeds reduce image quality and vice versa. In the scanning-based image collection methods, TF image collection time can vary considerably depending on the microscope conditions such as exposure time, the number of TF images needed, illumination source intensity, sample type, and method of scanning. All such factors must be optimized to achieve a suitable, low-noise image in the acceptable condition. Increasing the exposure time and the number of TF images will usually increase collection time, while increasing illumination source intensity decreases it due to lesser required exposure time. A sample that returns a large scattered intensity is beneficial in reducing the exposure time and hence $\mathrm{TF}$ image collection time. TF collection times of the scanning methods span from 
a relatively slow (in the order of minutes) to a relatively highspeed (in the order of less than a millisecond).

Scanning can either be performed continuously or using repeated, short, step-scans for the scanning-based TF image collection methods. In continuous-scanning approaches, the scanning part and the camera are synchronized that enables relatively high-speed TF image collections. For example, in the objective scanning method (Sec. 3.2), it is possible to continuously scan the objective back-and-forth and simultaneously record images at a relatively high-speed $\left(200 \mathrm{~ms}^{41}\right)$. The focus step size depends on the time gap between each exposure and the scanning speed of the objective. Even though the adaptive optics method (Sec. 3.8) is a type of continuous-scanning method, it achieves a very high-speed collection $\left(\approx 1 \mathrm{kHz}^{32}\right)$. Acoustooptic lens $(\approx 400 \mathrm{kHz}),{ }^{17}$ liquid lenses $(2 \mathrm{~ms} / 500 \mathrm{~Hz}),{ }^{11,93}$ and adaptive optics $(10 \mathrm{~ms} / 100 \mathrm{~Hz})^{66}$ can also achieve a high-speed TF image collection.

Step-scan methods are relatively slow as scanning must be stopped briefly for image collection at every step, and this increases the overall TF image collection time. For example, in the stage-scanning method (Sec. 3.1), each time the stage is axially translated at a given step size, it is stopped for image collection, repeating the process until all the TF images are collected.

Usually, nonscanning methods are relatively fast. For example, in MFM, TF images are collected simultaneously in one exposure (or in one image). This results in TF image collection as fast as any single image collection using a conventional microscope. Multifocal plane microscopy also can collect TF images simultaneously at a high-speed. However, the number of images is restricted to the number of times imaging beam is split. High-speed TF imaging can also be achieved using DHM. A summary of the best speeds possible to collect a set of TF images for different TF methods is presented in Table 1.

\subsection{Image Quality}

Not all TF imaging methods produce the same quality images. In fact, a wide range of image qualities can be expected depending on the TF method and the experimental conditions. It is the opinion of the author that the best quality images can be collected relatively easily using step-scan type of TF methods (e.g., stage-scanning method), but this is at the expense of collection speed. The presence of optical tool mechanical instability and other mechanical vibrations can deteriorate the image quality. In reality, scanning-based methods are usually prone to image deterioration of this kind. ${ }^{34}$ Consequently, if careful attention is not given to these factors, the continuous-scanning methods will usually result in lower quality images. Liquid-tunable lenses and flexible membrane liquid lenses suffer from significant aberrations ${ }^{56}$ which reduce image quality. Image quality in MFM can suffer because of splitting of the primary beam into several images.

As presented above, every TF method has certain advantages and disadvantages. The selection of a TF method depends on several factors, including type of data needed, image quality, applicability, speed of acquisition, cost, mechanical stability, accuracy, noise, simplicity, ease of use, and availability of the technology. Several aspects of optical microscopes have been identified to minimize degradation of optical images (e.g., laser stability, flat-field correction, camera performance, optical aberrations, noise, spectral reproducibility, lateral resolution, lens cleanliness, lens characteristics, temporal variability of signal and noise, absolute intensity calibration, and correcting field-dependent aberrations $\mathrm{s}^{90,94-102}$ ). However, to the best of our knowledge, there is no published method to test the fidelity of a set of TF images. Two aspects are uniquely associated with TF type of data collection: (i) focus step size and (ii) sample/stage vibration (or lateral movement) during mechanical scanning. Either imprecise focus step size (including in the extracted TF images) or lateral displacement of sample is highly likely to deteriorate TF optical data. A combination of (i) and (ii) could also be presented. For example, scanning methods could have mechanical instabilities and vibration issues ${ }^{34}$ resulting in increased overall noise. If there is a mismatch between the sample stage scan axis with the optical axis along the focus direction, TF optical images appear to shift laterally ${ }^{32}$ creating a large error. Similar lateral image shift can also be observed if the aperture diaphragm is not correctly aligned with the optical axis. ${ }^{20}$ For these reasons, it is important to evaluate fidelity of the TF data. We are actively working to develop a method to test TF data fidelity.

\section{Conclusion}

We have presented a review of over 15 distinct TF imaging methods from the literature. There is a wide choice to select from depending on the need. As with any optical images, the set of TF images collected using any method or under any conditions needs to be tested for fidelity. We hope to present such a test in the near future.

\section{Disclosure}

The author declares no conflict of interest.

\section{References}

1. S. W. Hell, "Far-field optical nanoscopy," in Single Molecule Spectroscopy in Chemistry, Physics and Biology: Nobel Symposium, A. Gräslund, R. Rigler, and J. Widengren, Eds., pp. 365-398, Springer, Berlin, Heidelberg (2010).

2. R. Zenobi, "Analytical tools for the nano world," Anal. Bioanal. Chem. 390(1), 215-221 (2008).

3. S. Doose, "Trends in biological optical microscopy," ChemPhysChem 9(4), 523-528 (2008).

4. L. Schermelleh, R. Heintzmann, and H. Leonhardt, "A guide to superresolution fluorescence microscopy," J. Cell Biol. 190(2), 165-175 (2010).

5. W. H. Stefan et al., "The 2015 super-resolution microscopy roadmap," J. Phys. D: Appl. Phys. 48(44), 443001 (2015).

6. M. J. Rust, M. Bates, and X. Zhuang, "Sub-diffraction-limit imaging by stochastic optical reconstruction microscopy (STORM)," Nat. Methods 3, 793-796 (2006).

7. E. Betzig et al., "Imaging intracellular fluorescent proteins at nanometer resolution," Science 313(5793), 1642-1645 (2006).

8. K. Fujita, "Follow-up review: recent progress in the development of super-resolution optical microscopy," Microscopy 65(4), 275-281 (2016).

9. A. von Diezmann, Y. Shechtman, and W. E. Moerner, "Three-dimensional localization of single molecules for super-resolution imaging and single-particle tracking," Chem. Rev. 117(11), 7244-7275 (2017).

10. A. Azaripour et al., "Three-dimensional histochemistry and imaging of human gingiva," Sci. Rep. U.K. 8(1), 1647 (2018).

11. G. Katona et al., "Fast two-photon in vivo imaging with three-dimensional random-access scanning in large tissue volumes," Nat. Methods 9, 201-208 (2012).

12. P. Prabhat et al., "Simultaneous imaging of different focal planes in fluorescence microscopy for the study of cellular dynamics in three dimensions," IEEE Trans. NanoBiosci. 3(4), 237-242 (2004). 
13. A. Tahmasbi et al., "Designing the focal plane spacing for multifocal plane microscopy," Opt. Express 22(14), 16706-16721 (2014).

14. W. Göbel, B. M. Kampa, and F. Helmchen, "Imaging cellular network dynamics in three dimensions using fast 3D laser scanning," Nat. Methods 4, 73-79 (2006).

15. K. F. Tehrani et al., "Fast axial scanning for 2-photon microscopy using liquid lens technology," Proc. SPIE 10070, 100700 Y (2017).

16. N. Ji, J. Freeman, and S. L. Smith, "Technologies for imaging neural activity in large volumes," Nat. Neurosci. 19, 1154-1164 (2016).

17. A. Kaplan, N. Friedman, and N. Davidson, "Acousto-optic lens with very fast focus scanning," Opt. Lett. 26(14), 1078-1080 (2001).

18. R. Attota et al., "Nanoparticle size determination using optical microscopes," Appl. Phys. Lett. 105(16), 163105 (2014).

19. H. Kang et al., "A method to determine the number of nanoparticles in a cluster using conventional optical microscopes," Appl. Phys. Lett. 107(10), 103106 (2015).

20. R. K. Attota and H. Park, "Optical microscope illumination analysis using through-focus scanning optical microscopy," Opt. Lett. 42(12), 2306-2309 (2017)

21. R. K. Attota et al., "Feasibility study on 3-D shape analysis of highaspect-ratio features using through-focus scanning optical microscopy," Opt. Express 24(15), 16574-16585 (2016).

22. R. K. Attota and H. Kang, "Parameter optimization for through-focus scanning optical microscopy," Opt. Express 24(13), 14915-14924 (2016).

23. R. Attota, "Noise analysis for through-focus scanning optical microscopy," Opt. Lett. 41(4), 745-748 (2016).

24. R. Attota and R. G. Dixson, "Resolving three-dimensional shape of sub-50 nm wide lines with nanometer-scale sensitivity using conventional optical microscopes," Appl. Phys. Lett. 105(4), 043101 (2014).

25. R. Attota, B. Bunday, and V. Vartanian, "Critical dimension metrology by through-focus scanning optical microscopy beyond the $22 \mathrm{~nm}$ node," Appl. Phys. Lett. 102(22), 222107 (2013).

26. A. Arceo, B. Bunday, and R. Attota, "Use of TSOM for sub-11 nm node pattern defect detection and HAR features," Proc. SPIE 8681, $86812 \mathrm{G}$ (2013).

27. A. Arceo et al., "Patterned defect and CD metrology by TSOM beyond the $22 \mathrm{~nm}$ node," Proc. SPIE 8324, 83240E (2012).

28. R. Attota and R. Silver, "Nanometrology using a through-focus scanning optical microscopy method," Meas. Sci. Technol. 22(2), 024002 (2011).

29. R. Attota et al., "TSOM method for semiconductor metrology," Proc. SPIE 7971, 79710T (2011).

30. R. Attota, T. A. Germer, and R. M. Silver, "Through-focus scanningoptical-microscope imaging method for nanoscale dimensional analysis," Opt. Lett. 33(17), 1990-1992 (2008).

31. S.-W. Park, J. H. Lee, and H. Kim, "3D digital holographic semiconductor metrology using Fourier modal method," in JSAP-OSA Joint Symp., p. 8a_PB2_1, OSA, Fukuoka, Japan (2017).

32. S. Han et al., "Tip/tilt-compensated through-focus scanning optical microscopy," Proc. SPIE 10023, 100230P (2016).

33. M. Ryabko et al., "Through-focus scanning optical microscopy (TSOM) considering optical aberrations: practical implementation," Opt. Express 23(25), 32215-32221 (2015).

34. M. Ryabko et al., "Motion-free all optical inspection system for nanoscale topology control," Opt. Express 22(12), 14958-14963 (2014).

35. S. Koptyaev, M. Ryabko, and M. Rychagov, "Optical measurement system and method for measuring critical dimension of nanostructure," Google Patents EP 2587313 B1 (2016).

36. S. Usha et al., "Through focus optical imaging technique to analyze variations in nano-scale indents," Int. J. Eng. Res. Technol. 2(5), 18 (2013).

37. M. V. Ryabko et al., "Method for optical inspection of nanoscale objects based upon analysis of their defocused images and features of its practical implementation," Opt. Express 21(21), 24483-24489 (2013).

38. S. Abrahamsson et al., "Fast multicolor 3D imaging using aberrationcorrected multifocus microscopy," Nat. Methods 10(1), 60-63 (2013).

39. S. Abrahamsson et al., "Multifocus microscopy with precise color multi-phase diffractive optics applied in functional neuronal imaging," Biomed. Opt. Express 7(3), 855-869 (2016).
40. S. Abrahamsson et al., "Multifocus polarization microscope (MFPolScope) for 3D polarization imaging of up to 25 focal planes simultaneously," Opt. Express 23(6), 7734-7754 (2015).

41. J. M. Gineste et al., "Three-dimensional automated nanoparticle tracking using Mie scattering in an optical microscope," J. Microsc. 243(2), 172-178 (2011).

42. M. Minsky, "Memoir on inventing the confocal scanning microscope," Scanning 10(4), 128-138 (1988).

43. W. E. Ortyn et al., "Extended depth of field imaging for high speed cell analysis," Cytom. Part A 71A(4), 215-231 (2007).

44. R. Attota et al., "Optical critical dimension measurement and illumination analysis using the through-focus focus metric: art. no. 61520K," Proc. SPIE 6152, K1520 (2006).

45. R. Attota et al., "Application of through-focus focus-metric analysis in high resolution optical metrology," Proc. SPIE 5752, 1441-1449 (2005).

46. R. M. Silver et al., "High-resolution optical overlay metrology," Proc. SPIE 5375, 78-95 (2004).

47. R. M. Silver et al., "Scatterfield microscopy for extending the limits of image-based optical metrology," Appl. Opt. 46(20), 4248-4257 (2007).

48. B. M. Barnes et al., "Optical volumetric inspection of sub-20 nm patterned defects with wafer noise," Proc. SPIE 9050, 905016 (2014).

49. R. K. Attota, "Step beyond Kohler illumination analysis for far-field quantitative imaging: angular illumination asymmetry (ANILAS) maps," Opt. Express 24(20), 22616-22627 (2016).

50. R. Attota and R. Silver, "Optical microscope angular illumination analysis," Opt. Express 20(6), 6693-6702 (2012).

51. E. A. Patterson and M. P. Whelan, "Optical signatures of small nanoparticles in a conventional microscope," Small 4(10), 1703-1706 (2008).

52. E. J. Botcherby et al., "An optical technique for remote focusing in microscopy," Opt. Commun. 281(4), 880-887 (2008).

53. A. Jesacher, C. Roider, and M. Ritsch-Marte, "Enhancing diffractive multi-plane microscopy using colored illumination," Opt Express 21(9), 11150-11161 (2013).

54. G. C. Knollman, J. L. S. Bellin, and J. L. Weaver, "Variable-focus liquid-filled hydroacoustic lens," J. Acoust. Soc. Am. 49(1), 253261 (1971).

55. C. Friese et al., "Materials, effects and components for tunable microoptics," IEEJ Trans. Electr. Electron. Eng. 2(3), 232-248 (2007).

56. P. P. Zhao, C. Ataman, and H. Zappe, "Miniaturized variable-focus objective employing a liquid-filled tunable aspherical lens," Opt. Eng. 56(10), 103110 (2017).

57. L. H. Wang, H. Oku, and M. Ishikawa, "Paraxial ray solution for liquid-filled variable focus lenses," Jpn. J. Appl. Phys. 56(12), 122501 (2017).

58. L. Wang et al., "Variable-focus liquid lens integrated with a planar electromagnetic actuator," Micromachines 7(10), 190 (2016).

59. W. X. Zhao et al., "Variable-focus cylindrical liquid lens array," Proc. SPIE 8769, 87690Y (2013).

60. P. Pokorný et al., "Deformation of a prestressed liquid lens membrane," Appl. Opt. 56(34), 9368-9376 (2017).

61. H. Ren and S.-T. Wu, "Variable-focus liquid lens," Opt. Express 15(10), 5931-5936 (2007).

62. J.-W. Du, X.-Y. Wang, and D. Liang, "Bionic optical imaging system with aspheric solid-liquid mixed variable-focus lens," Opt. Eng. 55, 023105 (2016).

63. H. Ren and S. T. Wu, "Variable-focus liquid lens by changing aperture," Appl. Phys. Lett. 86(21), 211107 (2005).

64. D. Koyama, R. Isago, and K. Nakamura, "Compact, high-speed variable-focus liquid lens using acoustic radiation force," Opt. Express 18(24), 25158-25169 (2010).

65. P. S. Salter, Z. Iqbal, and M. J. Booth, "Analysis of the three-dimensional focal positioning capability of adaptive optic elements," Int. J. Optomechatronics 7(1), 1-14 (2013).

66. M. Žurauskas et al., "Rapid adaptive remote focusing microscope for sensing of volumetric neural activity," Biomed. Opt. Express 8(10), 4369-4379 (2017).

67. S. Dong et al., "Aperture-scanning Fourier ptychography for 3D refocusing and super-resolution macroscopic imaging," Opt. Express 22(11), 13586-13599 (2014). 
68. T. F. Holekamp, D. Turaga, and T. E. Holy, "Fast three-dimensional fluorescence imaging of activity in neural populations by objectivecoupled planar illumination microscopy," Neuron 57(5), 661-672 (2008).

69. R. M. Power and J. Huisken, "A guide to light-sheet fluorescence microscopy for multiscale imaging," Nat. Methods 14, 360-373 (2017).

70. J. Huisken et al., "Optical sectioning deep inside live embryos by selective plane illumination microscopy," Science 305(5686), 10071009 (2004).

71. A.-K. Gustavsson et al., "3D single-molecule super-resolution microscopy with a tilted light sheet," Nat. Commun. 9(1), 123 (2018).

72. B. Weigelin, G.-J. Bakker, and P. Friedl, "Third harmonic generation microscopy of cells and tissue organization," J. Cell Sci. 129(2), 245255 (2016).

73. T. Lagerweij et al., "Optical clearing and fluorescence deep-tissue imaging for 3D quantitative analysis of the brain tumor microenvironment," Angiogenesis 20(4), 533-546 (2017).

74. M. Zhang et al., "Three-dimensional light field microscope based on a lenslet array," Opt. Commun. 403(Suppl. C), 133-142 (2017).

75. M. Levoy et al., "Light field microscopy," ACM Trans. Graphics 25(3), 924-934 (2006).

76. L. J. Allen and M. P. Oxley, "Phase retrieval from series of images obtained by defocus variation," Opt. Commun. 199(1), 65-75 (2001).

77. Z. Jingshan et al., "Transport of Intensity phase imaging by intensity spectrum fitting of exponentially spaced defocus planes," Opt. Express 22(9), 10661-10674 (2014).

78. B. Xue et al., "Transport of intensity phase imaging from multiple intensities measured in unequally-spaced planes," Opt. Express 19(21), 20244-20250 (2011).

79. G. Popescu, Quantitative Phase Imaging of Cells and Tissues, McGraw-Hill, New York (2011).

80. S. S. Kou and C. J. R. Sheppard, "Imaging in digital holographic microscopy," Opt. Express 15(21), 13640-13648 (2007).

81. M. Matrecano, M. Paturzo, and P. Ferraro, "Extended focus imaging in digital holographic microscopy: a review," Opt. Eng. 53, 112317 (2014).

82. T. Colomb et al., "Extended depth-of-focus by digital holographic microscopy," Opt. Lett. 35(11), 1840-1842 (2010).

83. J. P. Ryle et al., "Calibration of a digital in-line holographic microscopy system: depth of focus and bioprocess analysis," Appl. Opt. 52(7), C78-C87 (2013).

84. P. Ferraro et al., "Extended focused image in microscopy by digital holography," Opt. Express 13(18), 6738-6749 (2005).

85. J. Rosen and G. Brooker, "Fresnel incoherent correlation holography (FINCH): a review of research," in Advanced Optical Technologies, p. 151 (2012).
86. P. Ferraro et al., "Compensation of the inherent wave front curvature in digital holographic coherent microscopy for quantitative phasecontrast imaging," Appl. Opt. 42(11), 1938-1946 (2003).

87. M. E. Durst, G. Zhu, and C. Xu, "Simultaneous spatial and temporal focusing for axial scanning," Opt. Express 14(25), 12243-12254 (2006).

88. A. Dubois et al., "High-resolution full-field optical coherence tomography with a Linnik microscope," Appl. Opt. 41(4), 805-812 (2002).

89. E. Beaurepaire et al., "Full-field optical coherence microscopy," Opt. Lett. 23(4), 244-246 (1998).

90. R. M. Zucker and O. Price, "Evaluation of confocal microscopy system performance," Cytometry 44(4), 273-294 (2001).

91. T. J. Gould, S. T. Hess, and J. Bewersdorf, "Optical nanoscopy: from acquisition to analysis," Аnnu. Rev. Biomed. Eng. 14(1), 231-254 (2012).

92. F. Huang et al., "Ultra-high resolution 3D imaging of whole cells," Cell 166(4), 1028-1040 (2016).

93. H. Oku and M. Ishikawa, "Rapid liquid variable-focus lens with 2-ms response," in IEEE Lasers and Electro-Optics Society Annual Meeting-LEOS, p. 947 (2006).

94. R. Attota et al., "A new method to enhance overlay tool performance," Proc. SPIE 5038, 428-436 (2003).

95. R. M. Zucker, "Quality assessment of confocal microscopy slide-based systems: Instability," Cytom. Part A 69A(7), 677-690 (2006).

96. K. M. Kedziora et al., "Method of calibration of a fluorescence microscope for quantitative studies," J. Microsc. 244(1), 101-111 (2011).

97. J. C. Waters and T. Wittmann, "Quantitative Imaging in Cell Biology," in Methods in Cell Biology, 1st ed., Vol. 123, Elsevier (2014).

98. M. Halter et al., "An automated protocol for performance benchmarking a widefield fluorescence microscope," Cytom. Part A 85a(11), 978-985 (2014).

99. K. I. Mortensen and H. Flyvbjerg, "“Calibration-on-the-spot”: how to calibrate an EMCCD camera from its images," Sci. Rep. 7, 46883 (2016).

100. A. von Diezmann et al., "Correcting field-dependent aberrations with nanoscale accuracy in three-dimensional single-molecule localization microscopy," Optica 2(11), 985-993 (2015).

101. K. M. Douglass et al., "Super-resolution imaging of multiple cells by optimized flat-field epi-illumination," Nat. Photonics 10(11), 705-708 (2016).

102. F. Huang et al., "Video-rate nanoscopy using sCMOS camera-specific single-molecule localization algorithms," Nat. Methods 10(7), 653658 (2013).

Biographies for the authors are not available. 